Supporting information for:

\title{
Effective Wetting Area Based on Electrochemical Impedance Analysis:
}

\section{Hydrophilic Structured Surface}

\section{Dejian Zhang ${ }^{1 \dagger}$, Gyoko Nagayama ${ }^{2 \ddagger}$}

${ }^{1}$ Graduate School of Engineering, Kyushu Institute of Technology, Sensui 1-1, Tobata,

Kitakyushu, Fukuoka 804-8550, Japan

${ }^{2}$ Department of Mechanical Engineering, Kyushu Institute of Technology, Sensui 1-1,

Tobata, Kitakyushu, Fukuoka 804-8550, Japan

Corresponding Author:

Gyoko Nagayama

Email: nagayama.gyoko725@mail.kyutech.jp 


\section{Surface chemical composition analysis}

Typical X-ray diffraction (XRD) pattern obtained on the sample $\mathrm{f}$ is shown as Figure $\mathrm{S} 1$. The pattern is compared with the standard Al comparison card JCPDS No: 04-0787, and find the diffraction angle in 44.621, 64.906 and 78.102 are identical with $44.738,65.133$ and 78.227 of standard Al pattern (Figure S1). It is necessary to point out that after chemical etching, the copper layer formed on the surface have been purged up, whilst, no other new substance generated on the aluminum surface.

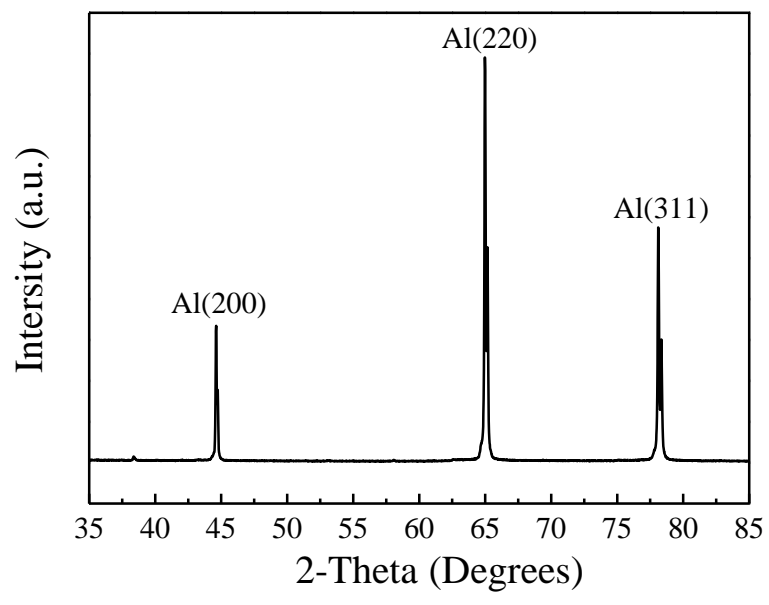

Figure S1. XRD patterns of the sample f.

\section{Contact angle measurement apparatus}

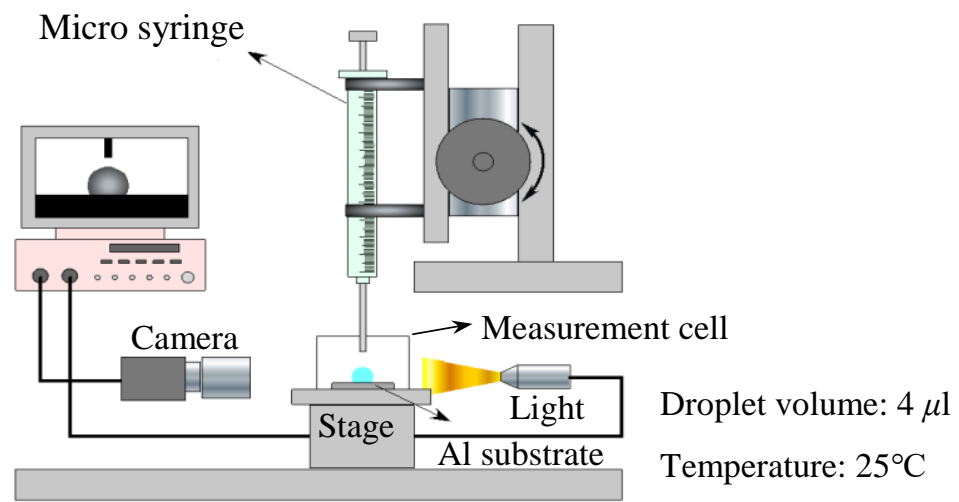

Figure S2. Contact angle measurement apparatus. 


\section{Confocal laser scanning microscope and cross section profile of samples}

Figure S3 shows confocal laser scanning microscopy (CLSM) images and cross-sectional profiles of samples (a)-(f). Each profiles below the images are the cross-section located at the yellow line shown in CLSM images.
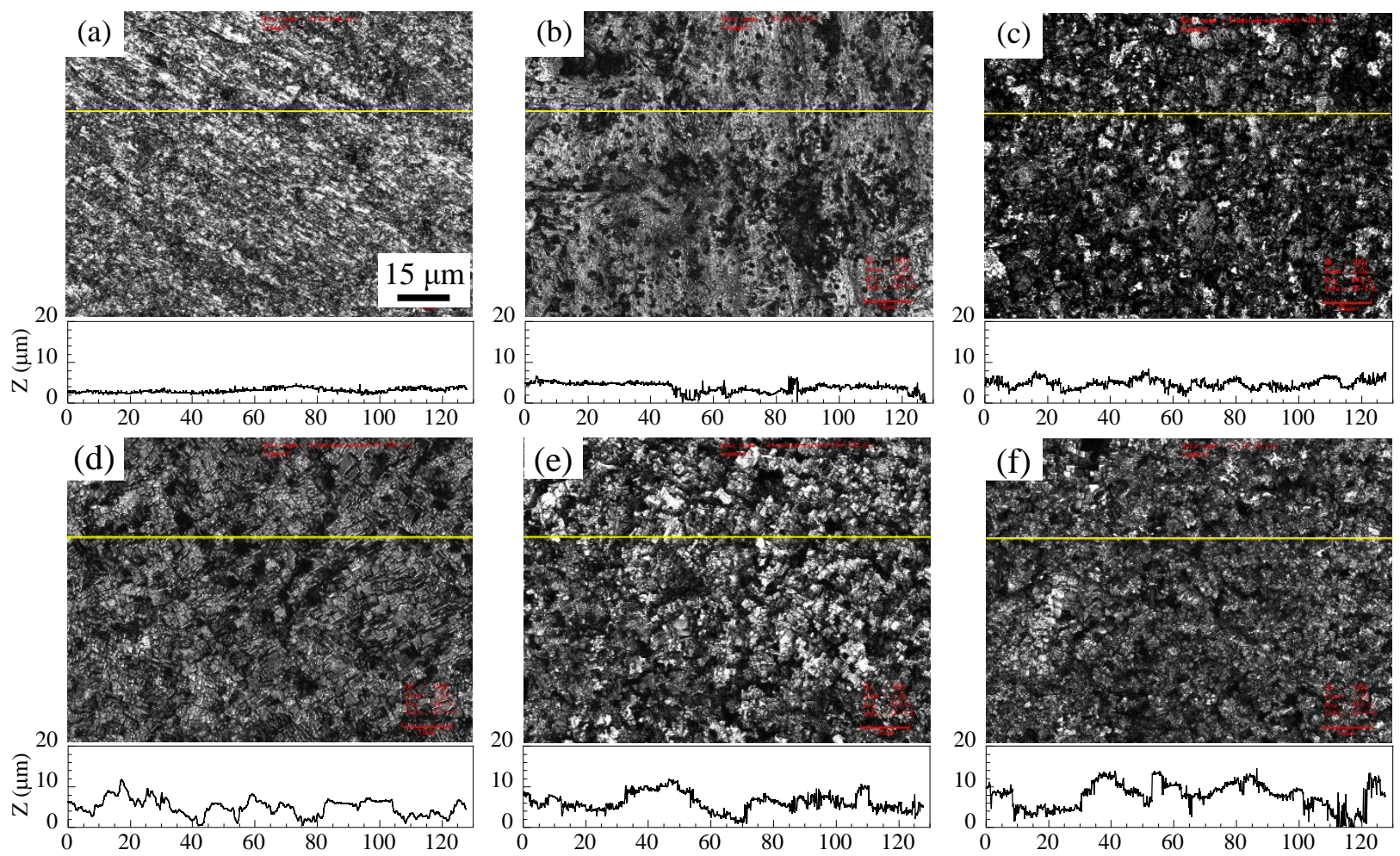

Figure S3. Confocal laser scanning microscopy images of sample surfaces and cross-sectional profiles after immersed in a $\mathrm{CuCl}_{2}$ solution for (a) 0 , (b) 1 , (c) 2, (d) 5 , (e) 10 , and (f) $20 \mathrm{~s}$. 
4. Comparisons of Nyquist plots simulated by CPE and $C_{\mathrm{dl}}$ for sturcutred surface.
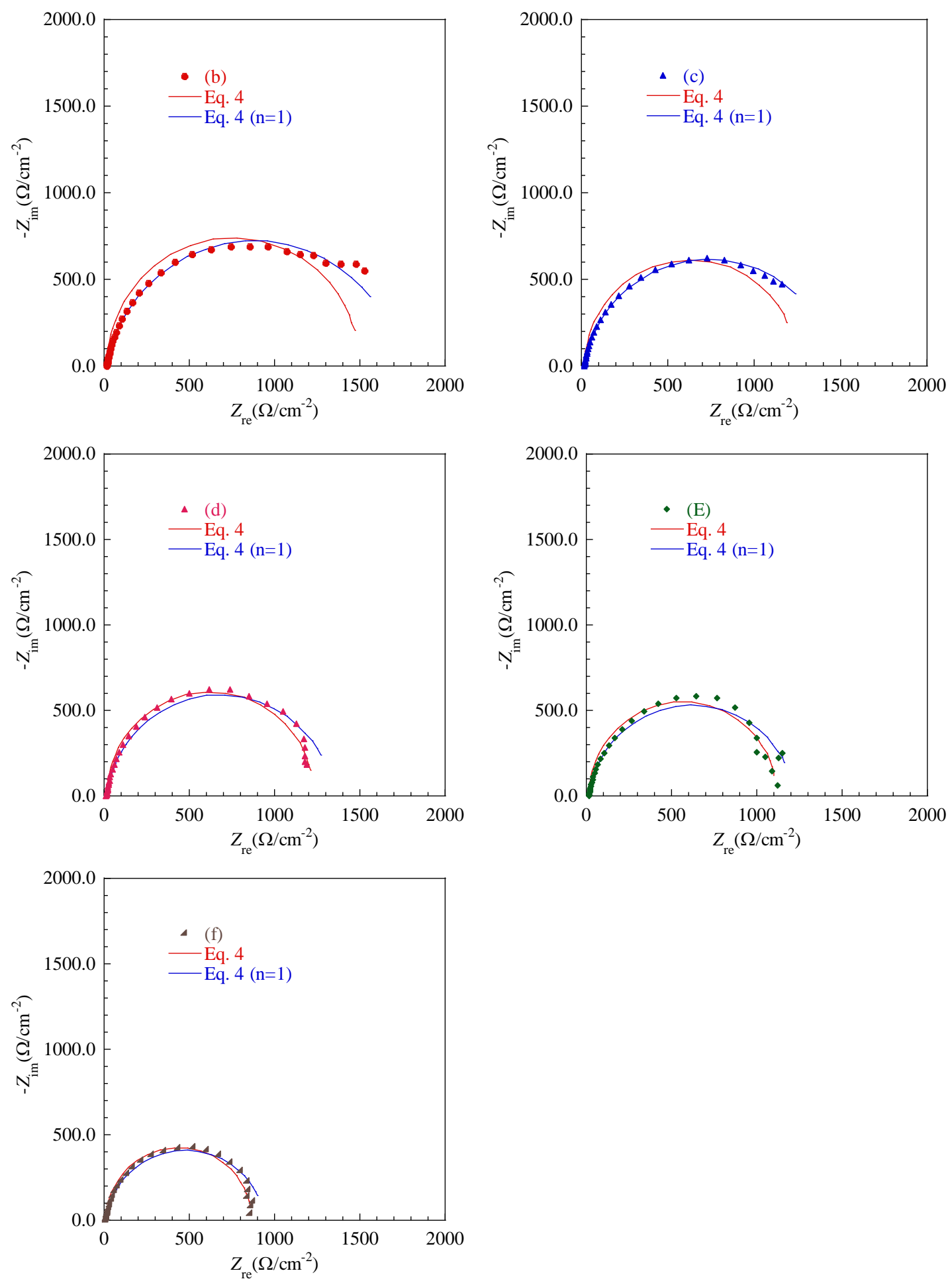

Figure S4. Nyquist plots of the experimental data and those derived from Eq. (4) for the sample (b)-(f). 


\section{Reason about impedance analysis at low frequency}

The reason we study the impedance at low frequency is because the impedance at low frequency indicate a better and clear electrochemical property of the substrates. At low frequency region (Figure S5 (a)), the wavelength of the disturb signal is long and the ions, electrons are hard to obtain energy which result in small penetration force. The charge only transfers at the solid-liquid contact area. On the other hand, the wavelength is short at high frequency region (Figure S5 (b)), ions and electrons are easy to obtain energy which result in large penetration force and no significant deviation of charge transfer at the solid-liquid interface and solid-vapor-liquid interface.

As a result, the solid-liquid contact area is estimated at the low frequency.

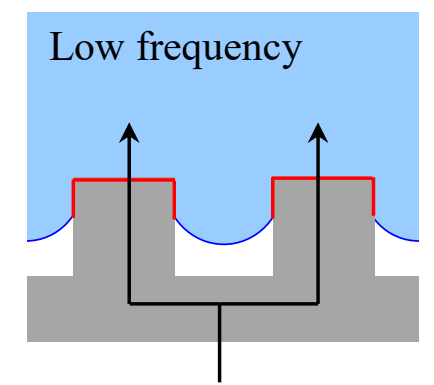

Long wavelength

Small energy

Small penetration force

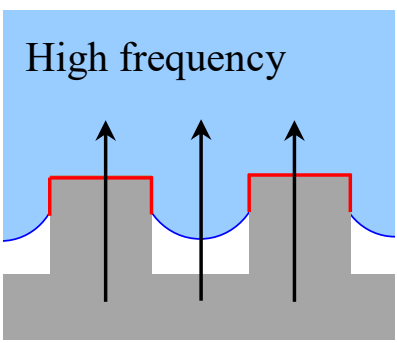

Short wavelength

Large energy

Large penetration force

Figure S5. Solid-liquid interface during electrochemical impedance measurement. (a) low frequency region, (b) high frequency region. 